\title{
SIMILAR CONSTRUCTING METHOD FOR SOLVING THE BOUNDARY VALUE PROBLEM OF THE COMPOSITE THOMSON EQUATION
}

\author{
Xiao-xu Dong ${ }^{1 *}$, Zhi-bin Liu' ${ }^{2}$ Duo Zhang ${ }^{3}$, Shun-chu $\mathrm{Li}^{4}$ \\ ${ }^{* 1,2}$ College of Science, Southwest Petroleum University, Chengdu, 610500, China \\ ${ }^{3,4}$ College of Science, Xihua University, Chengdu, 610039, China \\ 11ongxiaoxu1028@163.com, 2liuzhibinswpi@vip.sina.com, ${ }^{3}$ 15520453815@163.com ${ }^{4}$ lishunchu@163.com
}

Corresponding Author: -

Email:dongxiaoxu1028@163.com

\begin{abstract}
: -
Based on the theory of similar structure of solution of the boundary value problem of differential equation, this paper studies the solution of a class of boundary value problem of the composite Thomson equation, After analysis, it is indicate that the solution with a form of continued fraction product is obtained by introducing functions of guide solution, constructing similar kernel functions and assembling coefficients of the left non-homogeneous boundary condition, functions of guide solution and similar kernel functions in this paper. Then a new method is obtained for solving the composite boundary value problem_Similar Constructive Method. Furthermore, the function solution is clearly illustrated by the chart which is carried out by the corresponding program in MTLAB. Thus, the method is not only simple and effective for solving the complicated boundary value problem of differential equation, but also is a kind of innovation of ideological.
\end{abstract}

Keywords: -Boundary value problem, Composite Thomson equation, Similar Constructing Method, Similar kernel function, Function of guide solution. 


\section{INTRODUCTION}

The boundary value problem of differential equation in practical problems is applied more and more widely, especially in application of oil and gas well testing technology. The theory and interpretative method of well testing analysis has been developed rapidly and improved continuously. Therefore, the corresponding solving method of the boundary value problem of differential equation is also simultaneously required to be developed and improved. With the sustainable development and improvement of the theory of similar structure of solution of differential equations, its application in the field of engineering is also more widespread, and particularly it plays an active role in the solution to reservoir flow model.

At the beginning of this century, the thought of similar structure of the solution of the boundary value problem of differential equation began to form ${ }^{[1]}$. The theory of similar structure of the solution of the boundary value problem of differential equation was put forward. Meanwhile, a new method was obtained for solving the class boundary value problem of differential equation ${ }^{[2-16]}$. The new method is named Similar Constructing Method (shortened as SCM). The method is a convenient, effective and creative method for solving the boundary value problem of differential equation.

Based on the above study, this paper will study a class of boundary value problems of the composite Thomson equation as follows:

$$
\begin{cases}x^{2} y_{1}^{\prime \prime}+x y_{1}^{\prime}-\left(i x^{2}+v_{1}^{2}\right) y_{1}=0 & (a \leq x \leq c) \\ x^{2} y_{2}^{\prime \prime}+x y_{2}^{\prime}-\left(i x^{2}+v_{2}^{2}\right) y_{2}=0 & (c \leq x \leq b) \\ {\left[E y_{1}+(1+E F) y_{1}^{\prime}\right]_{x=a}=D} & \\ \left.y_{1}\right|_{x=c}=\left.\lambda y_{2}\right|_{x=c} \\ \left.y_{1}^{\prime}\right|_{x=c}=\left.\mu y_{2}^{\prime}\right|_{x=c} \\ {\left[M y_{2}+N y_{2}^{\prime}\right]_{x=b}=0}\end{cases}
$$

Here $D 、 E 、 F 、 M 、 N 、 a 、 b 、 c$ are constants $v_{j} \geq 0(j=1,2), D \neq 0, \lambda \mu \neq 0$, $M^{2}+N^{2} \neq 0,0<a<c<b, i^{2}=-1$. Furthermore, the function solution is clearly illustrated by the chart which is carried out by the corresponding program in MTLAB.

\section{PRELIMINARY KNOWLEDGE}

Lemma 1 With the variable substitutions $\zeta=\sqrt{i x}$, the first Weber equations $x^{2} y_{j}^{\prime \prime}+x y_{j}^{\prime}-\left(i x^{2}+v_{j}^{2}\right) y_{j}=0 \quad(j=1,2)$ can be transformed into modified Bessel equations as follows ${ }^{[17]}$.

$$
\zeta^{2} \frac{d^{2} y_{j}}{d \zeta^{2}}+\zeta \frac{d y_{j}}{d \zeta}-\left(\zeta^{2}+v_{j}^{2}\right) y_{j}=0 \quad(j=1,2)
$$

Proof By taking variable substitution $\zeta=\sqrt{i} x$ for the Thomson equation and calculating

$$
\begin{aligned}
& \text { first-order derivative and two-order derivative of } y_{i} \text { to } x \text { (i.e. } \\
& y_{j}^{\prime} \equiv \frac{d y_{j}}{d x}=\sqrt{i} \frac{d y_{j}}{d \zeta}, y_{j}^{\prime \prime} \equiv \frac{d y_{j}^{\prime}}{d x}=i \frac{d^{2} y_{j}}{d \zeta^{2}} \text {, the Thomson equation can be transformed into the }
\end{aligned}
$$

equation as follows:

$$
\zeta^{2} \frac{d^{2} y_{j}}{d \zeta^{2}}+\zeta \frac{d y_{j}}{d \zeta}-\left(\zeta^{2}+v_{j}^{2}\right) y_{j}=0 \quad(j=1,2) .
$$

Where, Eq. (2) is the modified Bessel equation ${ }^{[17]}$.

Lemma 2 General solution of the Thomson equation can be expressed as:

$$
y_{j}=A_{j} I_{v_{j}}(\sqrt{i} x)+B_{j} K_{v_{j}}(\sqrt{i} x) \quad(j=1,2)
$$

Here $A B_{i},{ }_{i}$ are arbitrarily real constants, and $I_{v_{j}}(\cdot), K_{v_{j}}(\cdot)$ are the first and the second class of modified Bessel functions of order $v_{j}{ }^{[17]}$.

Proof General solution of the modified Bessel equation can be expressed as:

$$
y_{j}=A_{j} I_{v_{j}}(\zeta)+B_{j} K_{v_{j}}(\zeta) \quad(j=1,2)
$$

According to the lemma 1 , we let $\zeta=\sqrt{i} x$, and then general solution of the Thomson equation can be obtained as follows:

$$
y_{j}=A_{j} I_{v_{j}}(\zeta)+B_{j} K_{v_{j}}(\zeta) \quad(j=1,2)
$$


Let

$$
\varphi_{m_{j}, n_{j}}(x, \xi, \sqrt{i})=K_{m_{j}}(\sqrt{i} x) I_{n_{j}}(\sqrt{i} \xi)+(-1)^{m_{j}-n_{j}+1} I_{m_{j}}(\sqrt{i} x) K_{n_{j}}(\sqrt{i} \xi) \quad(j=1,2)
$$

Lemma 3 If $I_{v_{j}}(\sqrt{i} x), K_{v_{j}}(\sqrt{i} x)$ are two linear independent solutions of the Thomson equations $x^{2} y_{j}^{\prime \prime}+x y_{j}^{\prime}-\left(i x^{2}+v_{j}^{2}\right) y_{j}=0$. We lead into functions of guide solution as follow:

$$
\varphi_{m, n}(x, \xi)=K_{m}(\sqrt{i} x) I_{n}(\sqrt{i} \xi)+(-1)^{m-n+1} I_{m}(\sqrt{i} x) K_{n}(\sqrt{i} \xi)
$$

Defining functions of guide solution as follows:

$$
\begin{aligned}
& \varphi_{0,0}^{j}(x, \xi) @ \varphi_{v_{j}, v_{j}}(x, \xi) \quad(j=1,2) \\
& \varphi_{1}^{j},(x, \xi) @ \frac{\partial}{\partial x} \varphi_{v_{j} v_{j}}(x, \xi)=\frac{v_{j}}{x} \varphi_{v},(x \xi)-\sqrt{\varphi_{v+j}} \|_{, j}, \quad\left(\begin{array}{ll}
=j & 1
\end{array}\right) \\
& \varphi_{0,1}^{j}(x, \xi) @ \frac{\partial}{\partial \xi} \varphi_{v_{j}, v_{j}}(x, \xi)=\frac{v_{j}}{\xi} \varphi_{v_{j}, v_{j}}(x, \xi)+\sqrt{i} \varphi_{v_{j}, v_{j}+1}(x, \xi) \quad(j=1,2) \\
& \varphi_{1,1}^{j}(x, \xi) @ \frac{\partial^{2}}{\partial x \partial \xi} \varphi_{v_{j}, v_{j}}(x, \xi)=\frac{v_{j}^{2}}{x \xi} \varphi_{v_{j}, v_{j}}(x, \xi)-\frac{\sqrt{i} v_{j}}{\xi} \varphi_{v_{j}+1, v_{j}}(x, \xi) \\
& +\frac{\sqrt{i v_{j}}}{x} \varphi_{v_{j}, v_{j}+1}(x, \xi)-i \varphi_{v_{j}+1, v_{j}+1}(x, \xi) \quad(j=1,2)
\end{aligned}
$$

Here $j=1$ denotes left region $(a \leq x \leq c), j=2$ denotes right region $(c \leq x \leq b)$.

\section{THE MAIN THEOREM AND ITS PROOF}

Theorem If the boundary value problem (1) has unique solution, then the solution of left region is expressed as:

$$
y_{1}=D \cdot \frac{1}{E+\frac{1}{F+\Phi_{1}(a)}} \cdot \frac{1}{F+\Phi_{1}(a)} \cdot \Phi_{1}(x) \quad(a \leq x \leq c)
$$

and the solution of right region is expressed as:

$$
y_{2}=D \cdot \frac{1}{E+\frac{1}{F+\Phi_{1}(a)}} \cdot \frac{1}{F+\Phi_{1}(a)} \cdot \frac{\varphi_{0,1}^{1}(c, c)}{\lambda \Phi_{2}(c) \varphi_{1,1}^{1}(a, c)-\mu \varphi_{1,0}^{1}(a, c)} \cdot \Phi_{2}(x) \quad(c \leq x \leq b),
$$

Here $\Phi_{2}(x)$ is called the similar kernel function of right region:

$$
\Phi_{2}(x)=\frac{M \varphi_{0,0}^{2}(x, b)+N \varphi_{0,1}^{2}(x, b)}{M \varphi_{1,0}^{2}(c, b)+N \varphi_{1,1}^{2}(c, b)} \quad(c \leq x \leq b),
$$

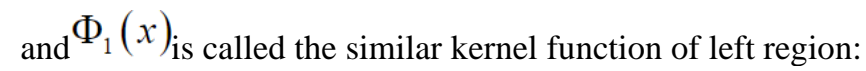

$$
\Phi_{1}(x)=\frac{\lambda \Phi_{2}(c) \varphi_{0,1}^{1}(x, c)-\mu \varphi_{0,0}^{1}(x, c)}{\lambda \Phi_{2}(c) \varphi_{1,1}^{1}(a, c)-\mu \varphi_{1,0}^{1}(a, c)} \quad(c \leq x \leq b)
$$

Proof According to the lemma 2, we know that general solutions of governing equations of left and right region of the boundary value problem (1) are

$$
y_{j}(x)=A_{j} I_{v_{j}}(\sqrt{i} x)+B_{j} K_{v_{j}}(\sqrt{i} x) \quad(j=1,2)
$$

Thus

$$
\begin{aligned}
y_{j}^{\prime}(x) & =\frac{d}{d x}\left[A_{j} I_{v_{j}}(\sqrt{i x})+B_{j} K_{v_{j}}(\sqrt{i x})\right] \\
& =A_{j}\left[\frac{v_{j}}{x} I_{v_{j}}(\sqrt{i x})+\sqrt{i} I_{v_{j}+1}(\sqrt{i x})\right]+B_{j}\left[\frac{v_{j}}{x} K_{v_{j}}(\sqrt{i x})-\sqrt{i} K_{v_{j}+1}(\sqrt{i x})\right] .
\end{aligned}
$$

By substituting Eqs.(13) and (14) into left and right boundary conditions and two connection conditions of the boundary value problem (1), we obtain the following equations respectively: 


$$
\begin{aligned}
& A_{1}\left\{E I_{v_{1}}(i a)+(1+E F)\left[\frac{v_{1}}{a} I_{v_{1}}(\sqrt{i} a)+\sqrt{i} I_{v_{1}+1}(\sqrt{i} a)\right]\right\} \\
& +B_{1}\left\{E K_{v_{1}}(\sqrt{i} a)+(1+E F)\left[\frac{v_{1}}{a} K_{v_{1}}(\sqrt{i} a)-\sqrt{i} K_{v_{1}+1}(\sqrt{i} a)\right]\right\}=D \\
& A_{1} I_{v_{1}}(\sqrt{i c})+B_{1} K_{v_{1}}(\sqrt{i c})-A_{2} \lambda I_{v_{2}}(\sqrt{i c})-B_{2} \lambda K_{v_{2}}(\sqrt{i c})=0 \\
& A_{1}\left[\frac{v_{1}}{c} I_{v_{1}}(\sqrt{i c})+\sqrt{i} I_{v_{1}+1}(\sqrt{i c})\right]+B_{1}\left[\frac{v_{1}}{c} K_{v_{1}}(\sqrt{i c})-\sqrt{i} K_{v_{1}+1}(\sqrt{i c})\right] \\
& -A_{2} \mu\left[\frac{v_{2}}{c} I_{v_{2}}(\sqrt{i c})+\sqrt{i} I_{v_{2}+1}(\sqrt{i c})\right]-B_{2} \mu\left[\frac{v_{2}}{c} K_{v_{2}}(\sqrt{i c})-\sqrt{i} K_{v_{2}+1}(\sqrt{i c})\right]=0 \\
& A_{2}\left\{M I_{v_{2}}(\sqrt{i b})+N\left[\frac{v_{2}}{b} I_{v_{2}}(\sqrt{i} b)+\sqrt{i} I_{v_{2}+1}(\sqrt{i} b)\right]\right\} \\
& +B_{2}\left\{M K_{v_{2}}(\sqrt{i} b)+N\left[\frac{v_{2}}{b} K_{v_{2}}(\sqrt{i} b)-\sqrt{i} K_{v_{2}+1}(\sqrt{i} b)\right]\right\}=0
\end{aligned}
$$

According to Eqs.(5) (8), we know that the coefficient determinant $\Delta$ of linear equation (Eqs.(15) (18)) about undetermined coefficients is not equal to zero, and

$$
\begin{aligned}
\Delta= & E\left\{-\mu \varphi_{0,0}^{1}(a, c)\left[M \varphi_{1,0}^{2}(c, b)+N \varphi_{1,1}^{2}(c, b)\right]+\lambda \varphi_{0,1}^{1}(a, c)\left[M \varphi_{0,0}^{2}(c, b)+N \varphi_{0,1}^{2}(c, b)\right]\right\} \\
& +(1+E F)\left\{-\mu \varphi_{1,0}^{1}(a, c)\left[M \varphi_{1,0}^{2}(c, b)+N \varphi_{1,1}^{2}(c, b)\right]+\lambda \varphi_{1,1}^{1}(a, c)\left[M \varphi_{0,0}^{2}(c, b)+N \varphi_{0,1}^{2}(c, b)\right]\right\}
\end{aligned}
$$

Values of $A B A B_{1}, 1,2,2$ can be obtained by using the Cramer rule as follows:

$$
\begin{aligned}
& A_{1}=\frac{D}{\Delta}\left\{\mu K_{v_{1}}(\sqrt{i c})\left[M \varphi_{1,0}^{2}(c, b)+N \varphi_{1,1}^{2}(c, b)\right]-\lambda\left[\frac{v_{1}}{c} K_{v_{1}}(\sqrt{i c})-\sqrt{i} K_{v_{1}+1}(\sqrt{i c})\right]\right. \\
& \left.\cdot\left[M \varphi_{0,0}^{2}(c, b)+N \varphi_{0,1}^{2}(c, b)\right]\right\} \\
& B_{1}=-\frac{D}{\Delta}\left\{\mu I_{v_{1}}(\sqrt{i c})\left[M \varphi_{1,0}^{2}(c, b)+N \varphi_{1,1}^{2}(c, b)\right]-\lambda\left[\frac{v_{1}}{c} I_{v_{1}}(\sqrt{i c})+\sqrt{i} I_{v_{+1}}(\sqrt{i c})\right]\right. \\
& \left.\cdot\left[M \varphi_{0,0}^{2}(c, b)+N \varphi_{0,1}^{2}(c, b)\right]\right\} \\
& A_{2}=-\frac{D}{\Delta}\left\{M K_{v_{2}}(\sqrt{i b})+N\left[\frac{v_{2}}{b} K_{v_{2}}(\sqrt{i b})-\sqrt{i} K_{\text {出 } 1}(\sqrt{i b})\right]\right\} \cdot \varphi_{0}^{1}(\varphi, c) \\
& B_{2}=\frac{D}{\Delta}\left\{M I_{v_{2}}(\sqrt{i b})+N\left[\frac{v_{2}}{b} I_{v_{2}}(\sqrt{i b})+\sqrt{i} I_{\text {H }_{2} 1}(\sqrt{i b})\right]\right\} \cdot \varphi_{0}^{1}(\varphi, c)
\end{aligned}
$$

By substituting values of $A B A B_{1,1,2,2}$ (Eqs.(20) (23))into Eq.(13) and using the similar kernel function of right region Eq.(11) and the similar kernel function of left region Eq.(12), solutions of left and right regions of the boundary value problem (1) are obtained respectively. i.e. Eq.(9) and Eq.(10).

Corollary 1 In the boundary value problem (1), if the right boundary condition is $y_{2}(b)=0$ (i.e. $M \neq 0, N=0$ ) or $y_{2}^{\prime}(b)=0$ (i.e. $M=0, N \neq 0$ ), the corresponding similar kernel function of peripheral region is

$$
\Phi_{2}(x)=\varphi_{0,0}^{2}(x, b) / \varphi_{1,0}^{2}(c, b) \text { or } \Phi_{2}(x)=\varphi_{0,1}^{2}(x, b) / \varphi_{1,1}^{2}(c, b) .
$$

Corollary 2 In the boundary value problem (1), if the left boundary condition is the second boundary condition (i.e. $\left.y_{1}^{\prime}\right|_{x=a}=1$ ), region (i.e. $\Phi_{1}(x)$ ). 
Corollary 3 The first continued fraction, which belongs to the structure of the solution (i.e. Eq.

(8)) of the boundary value problem (1), has the following property:

$$
\left[y_{1}(x)+F y_{1}^{\prime}(x)\right]_{x=a}=\frac{D}{E+\frac{1}{F+\Phi_{1}(a)}} .
$$

\section{STEPS OF THE SCM}

According to the proof of lemma 2 and theorem 1, it is easy to induce steps of the SCM for solving the boundary value problem of the composite first Weber equation. The concrete steps are as follows:

Step 1 Constructing functions of guide solution we structure the function of guide solution of left and right region by using two linear independent solutions $I_{v_{j}}(\sqrt{i} x)$ and $K_{v_{j}}(\sqrt{i} x)(j=1,2)$ of the governing equation of the left and right region of the boundary value problem (1) as follows: $\varphi_{v_{j}, v_{j}}(x, \xi)(j=1,2)$. Other functions of guide solution can be obtained by calculating partial derivatives of $\varphi_{v_{j}, v_{j}}(x, \xi)(j=1,2)$ to $x, \xi$ respectively.

\section{Step 2 Constructing similar kernel functions of left and right regions}

The similar kernel function $\Phi_{2}(x)$ of right region of the boundary value problem (1) can be structured by using functions of guide solution of right region $\varphi_{s, t}^{2}(x, \xi)(s, t=0,1)$ and coefficients $M, N$ of the homogeneous right boundary condition, as shown Eq.(11). Further we calculate $\Phi_{2}(c)$. The similar kernel function $\Phi_{1}(x)$ of left region of the boundary value problem (1) can be structured by using functions of guide solution of left ${ }_{\text {region }} \varphi_{s, t}^{1}(x, \xi)(s, t=0,1)$, coefficients $\lambda, \mu$ of two connection conditions and $\Phi_{2}(c)$, as shown Eq.(12). Further we calculate $\Phi_{1}(a)$

\section{Step 3 Obtaining solutions of the boundary value problem}

To the boundary value problem (1), the solution of the left region can be obtained by assembling coefficients $D, E, F$ of the non-homogeneous left boundary condition, the similar kernel function $\Phi_{1}(x)_{\text {of left region and }} \Phi_{1}(a)$, as shown Eq.(9). The solution of the right region can be obtained by assembling the coefficients $D, E, F$ of the non-homogeneous left boundary condition, the function of guide solution of left region $\varphi_{s, t}^{1}(x, \xi)(s, t=0,1)$, coefficients $\lambda, \mu$ of two connection conditions, the similar kernel function $\Phi_{2}(x)$ of right region, $\Phi_{2}(c)$ and $\Phi_{1}(a)$, as shown Eq.(10).

\section{THE APPLICATION OF THE SCM}

Solving the boundary value problem as follows:

$$
\left\{\begin{array}{l}
x^{2} y_{1}^{\prime \prime}+x y_{1}^{\prime}-i x^{2} y_{1}=0 \quad(1 \leq x \leq 4) \\
x^{2} y_{2}^{\prime \prime}+x y_{2}^{\prime}-\left(i x^{2}+1\right) y_{2}=0 \quad(4 \leq x \leq 10) \\
\left.y_{1}^{\prime}\right|_{x=1}=1 \\
\left.y_{1}\right|_{x=4}=\left.y_{2}\right|_{x=4} \\
\left.y_{1}^{\prime}\right|_{x=4}=\left.2 y_{2}^{\prime}\right|_{x=4} \\
\left.y_{2}^{\prime}\right|_{x=10}=0
\end{array}\right.
$$

Comparing with the boundary value problem (1) and (25), we know that $v_{1}=0, v_{2}=1, a=1$, $b=10, \quad c=4, \lambda=1, \quad \mu=2, D=1, E=0, M=0, N=1$. According to steps of the SCM, we solve the boundary value problem (25). 


\section{Step 1 Constructing functions of guide solution}

According to Eqs.(5) (8), we structure functions of guide solution of left and right regions as follows:

$$
\begin{aligned}
& \varphi_{0,0}^{1}(x, \xi)=K_{0}(\sqrt{i} x) I_{0}(\sqrt{i} \xi)-I_{0}(\sqrt{i} x) K_{0}(\sqrt{i} \xi) \\
& \varphi_{0,1}^{1}(x, \xi)=\sqrt{i}\left[K_{0}(\sqrt{i} x) I_{1}(\sqrt{i} \xi)+I_{0}(\sqrt{i} x) K_{1}(\sqrt{i} \xi)\right] \\
& \varphi_{1,0}^{1}(x, \xi)=-\sqrt{i}\left[K_{1}(\sqrt{i} x) I_{0}(\sqrt{i} \xi)+I_{1}(\sqrt{i} x) K_{0}(\sqrt{i} \xi)\right] \\
& \varphi_{1,1}^{1}(x, \xi)=-i\left[K_{1}(\sqrt{i x}) I_{1}(\sqrt{i} \xi)-I_{1}(\sqrt{i x}) K_{1}(\sqrt{i} \xi)\right] \\
& \varphi_{0,0}^{2}(x, \xi)=K_{1}(\sqrt{i} x) I_{1}(\sqrt{i} \xi)-I_{1}(\sqrt{i x}) K_{1}(\sqrt{i} \xi) \\
& \varphi_{0,1}^{2}(x, \xi)=\frac{1}{\xi}\left[K_{1}(\sqrt{i} x) I_{1}(\sqrt{i} \xi)-I_{1}(\sqrt{i} x) K_{1}(\sqrt{i} \xi)\right] \\
& +\sqrt{i}\left[K_{1}(\sqrt{i} x) I_{2}(\sqrt{i} \xi)+I_{1}(\sqrt{i} x) K_{2}(\sqrt{i} \xi)\right] \\
& \varphi_{1,0}^{2}(x, \xi)=\frac{1}{x}\left[K_{1}(\sqrt{i} x) I_{1}(\sqrt{i} \xi)-I_{1}(\sqrt{i} x) K_{1}(\sqrt{i} \xi)\right] \\
& -\sqrt{i}\left[K_{2}(\sqrt{i} x) I_{1}(\sqrt{i} \xi)+I_{2}(\sqrt{i} x) K_{1}(\sqrt{i} \xi)\right] \\
& \varphi_{1,1}^{2}(x, \xi)=\frac{1}{x \xi}\left[K_{1}(\sqrt{i} x) I_{1}(\sqrt{i} \xi)-I_{1}(\sqrt{i} x) K_{1}(\sqrt{i} \xi)\right]-\frac{\sqrt{i}}{\xi}\left[K_{2}(\sqrt{i} x) I_{1}(\sqrt{i} \xi)\right. \\
& \left.+I_{2}(\sqrt{i} x) K_{1}(\sqrt{i} \xi)\right]+\frac{\sqrt{i}}{x}\left[K_{1}(\sqrt{i} x) I_{2}(\sqrt{i} \xi)+I_{1}(\sqrt{i} x) K_{2}(\sqrt{i} \xi)\right] \\
& -i\left[K_{2}(\sqrt{i} x) I_{2}(\sqrt{i} \xi)-I_{2}(\sqrt{i} x) K_{2}(\sqrt{i} \xi)\right]
\end{aligned}
$$

\section{Step 2 Constructing similar kernel functions of left and right regions}

According to the Eq.(11), we structure the similar kernel function of right region of the boundary value problem (25) by using Eqs.(7) (8) as follows:

$$
\begin{aligned}
\Phi_{2}(x) & =\left[\frac{1}{10} K_{1}(\sqrt{i x}) I_{1}(10 \sqrt{i})-\frac{1}{10} I_{1}(\sqrt{i x}) K_{1}(10 \sqrt{i})+\sqrt{i} K_{1}(\sqrt{i x}) I_{2}(10 \sqrt{i})\right. \\
& \left.+\sqrt{i} I_{1}(\sqrt{i x}) K_{2}(10 \sqrt{i})\right] \times\left[\frac{1}{40} K_{1}(4 \sqrt{i}) I_{1}(10 \sqrt{i})-\frac{1}{40} I_{1}(4 \sqrt{i}) K_{1}(10 \sqrt{i})\right. \\
& -\frac{\sqrt{i}}{10} K_{2}(4 \sqrt{i}) I_{1}(10 \sqrt{i})-\frac{\sqrt{i}}{10} I_{2}(4 \sqrt{i}) K_{1}(10 \sqrt{i})+\frac{\sqrt{i}}{4} K_{1}(4 \sqrt{i}) I_{2}(10 \sqrt{i}) . \\
& \left.+\frac{\sqrt{i}}{4} I_{1}(4 \sqrt{i}) K_{2}(10 \sqrt{i})-i K_{2}(4 \sqrt{i}) I_{2}(10 \sqrt{i})+i I_{2}(4 \sqrt{i}) K_{2}(10 \sqrt{i})\right]^{-1} \\
& (4 \leq x \leq 10) \\
\Phi_{2}(4)= & {\left[\frac{1}{10} K_{1}(4 \sqrt{i}) I_{1}(10 \sqrt{i})-\frac{1}{10} I_{1}(4 \sqrt{i}) K_{1}(10 \sqrt{i})+\sqrt{i} K_{1}(4 \sqrt{i}) I_{2}(10 \sqrt{i})\right.} \\
& \left.+\sqrt{i} I_{1}(4 \sqrt{i}) K_{2}(10 \sqrt{i})\right] \times\left[\frac{1}{40} K_{1}(4 \sqrt{i}) I_{1}(10 \sqrt{i})-\frac{1}{40} I_{1}(4 \sqrt{i}) K_{1}(10 \sqrt{i})\right. \\
& -\frac{\sqrt{i}}{10} K_{2}(4 \sqrt{i}) I_{1}(10 \sqrt{i})-\frac{\sqrt{i}}{10} I_{2}(4 \sqrt{i}) K_{1}(10 \sqrt{i})+\frac{\sqrt{i}}{4} K_{1}(4 \sqrt{i}) I_{2}(10 \sqrt{i}) \\
& \left.+\frac{\sqrt{i}}{4} I_{1}(4 \sqrt{i}) K_{2}(10 \sqrt{i})-i K_{2}(4 \sqrt{i}) I_{2}(10 \sqrt{i})+i I_{2}(4 \sqrt{i}) K_{2}(10 \sqrt{i})\right]^{-1}
\end{aligned}
$$




$$
\begin{aligned}
\Phi_{1}(x) & =\left\{\Phi_{2}(4)\left[\sqrt{i} K_{0}(\sqrt{i x}) I_{1}(4 \sqrt{i})+\sqrt{i} I_{0}(\sqrt{i} x) K_{1}(4 \sqrt{i})\right]-2 K_{0}(\sqrt{i x}) I_{0}(4 \sqrt{i})\right. \\
& \left.+2 I_{0}(\sqrt{i} x) K_{0}(4 \sqrt{i})\right\} \times\left\{\Phi_{2}(4)\left[-i K_{1}(\sqrt{i}) I_{1}(4 \sqrt{i})+i I_{1}(\sqrt{i}) K_{1}(4 \sqrt{i})\right],\right. \\
& \left.+2 \sqrt{i} K_{1}(\sqrt{i}) I_{0}(4 \sqrt{i})+2 \sqrt{i} I_{1}(\sqrt{i}) K_{0}(4 \sqrt{i})\right\}^{-1} \quad(1 \leq x \leq 4)
\end{aligned}
$$

Step 3 Obtaining solutions of the boundary value problem (24)

According to Eqs.(9) and (10), solutions of left and right regions of the boundary value problem (25) can be obtained respectively as follows:

$$
\begin{aligned}
y_{1}(x)= & \Phi_{1}(x) \quad(1 \leq x \leq 4), \\
y_{2}(x)= & {\left[\sqrt{i} K_{0}(4 \sqrt{i}) I_{1}(4 \sqrt{i})+\sqrt{i} I_{0}(4 \sqrt{i}) K_{1}(4 \sqrt{i})\right] \cdot \Phi_{2}(x) \cdot\left\{-\Phi_{2}(4)\left[i K_{1}(\sqrt{i})\right.\right.} \\
\cdot I_{1} & \left.\left.(4 \sqrt{i})-i I_{1}(\sqrt{i}) K_{1}(4 \sqrt{i})\right]+2 \sqrt{i} K_{1}(\sqrt{i}) I_{0}(4 \sqrt{i})+2 \sqrt{i} I_{1}(\sqrt{i}) K_{0}(4 \sqrt{i})\right\}^{-1} . \\
& (4 \leq x \leq 10)
\end{aligned}
$$

According to above steps, we write a program by using MATLAB language. Then we can draw the graph of the solution of the boundary value problem (25) as follows(figure 1):

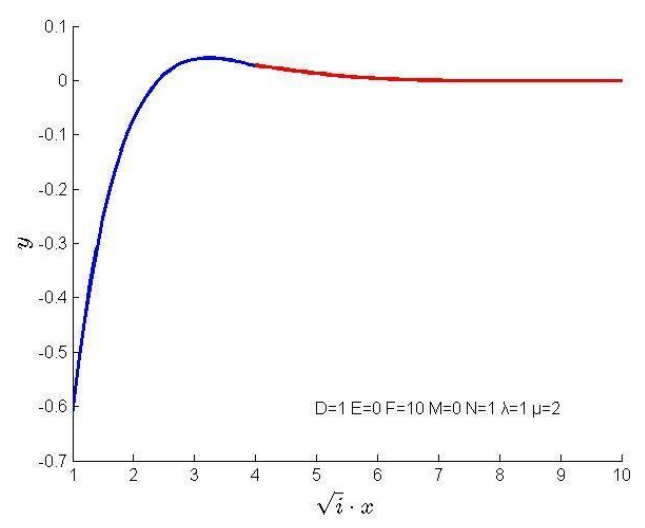

Figure 1: $y \sim \sqrt{i x}$ diagram

\section{CONCLUSIONS}

(1) In the process of solving the boundary value problem of the composite Thomson equation, we just need to obtain two linear independent solutions of governing equation of left region and two linear independent solutions of governing equation of right region of the boundary value problem respectively. Then according to Steps of the SCM, we can obtain solutions of the boundary value problem. Thus, using SCM can avoid the tedious calculation process.

(2) According to structural equations of similar kernel functions Eqs. (11) and (12) and structural equations of solutions of the boundary value problem (1) Eqs. (9) and (10), we know that we only need to change coefficients of boundary conditions to obtain solutions of the boundary value problem (1), when boundary conditions of the boundary value problem (1) change. Thus, Similar Constructing Method is simple and effective for solving the complicated boundary value problem of differential equation.

(3) Using MATLAB language to compile the program, we can draw the curve of the solution of the boundary value problem (25). It can verify the method of SCM is correct for solving the boundary value problem of composite Thomson equation.

\section{ACKNOWLEDGMENT}

This Project is supported by the scientific project of Sichuan provincial education department (No.15ZB0447).

\section{REFERENCES}

[1]. Li S. C., Preliminary Exploration and Prospects of the Similar Structure of Solutions of Differential Equations (in Chinese) [J].Journal of Xihua University (Nature Science Edition). 2010,29(2):223-226(to 238).

[2]. Li S. C., Yi L. Z., Zheng P. S., The Similar Structure of Differential Equations on Fixed Solution Problem (in Chinese) [J]. Journal of Sichuan University (Natural Science Edition), 2006,43(4):933 934.

[3]. Xu C. X., Li S. C., Zhu W. B., et al. The Similar Structure of Pressure Distribution in the Dual Porosity Reservoir (in Chinese) [J]. Drilling \& Production Technology,2006,29(4):28 30.

[4]. Li S. C., The Similar Structure of Solution of Second-order Linear Homogeneous Differential Equations with Constant Coefficients on the Boundary Value Problem (in Chinese) [J]. Journal of Xihua University (Natural Science Edition), 2007,26(1):84 85. 
[5]. Zheng P. S., Li S. C., Xu W.Z., Well Analysis Method Based on the Similar Structure of Pressure Distribution in the Composite Reservoir [J]. Drilling \& Production Technology,2007,30(3): 49 50(-62).

[6]. Li S. C., Zheng P. S, Zhang Y. F., The Similar Structure of Pressure Distribution in the Composite Reservoir (in Chinese) [J]. Journal of Mathematics in Practice and Theory, 2008,38(3):23 28.

[7]. Zhu W.B., Li S. C., Xu C.X., The Similar Structure of Pressure Distribution in the Fractal Multilayer Reservoir (in Chinese) [J]. Drilling \& Production Technology,2008,31(3):67 69(-72).

[8]. Li S. C., The Similar Structure of Solution to the Boundary Value Problem for Second-order Linear Homogeneous Differential Equations (in Chinese) [J]. Journal of Xihua University (Natural Science Edition), 2009,28(5): 40 41(to 90).

[9]. Li S. C., Preliminary Exploration and Prospects of the Similar Structure of Solutions of Differential Equations (in Chinese) [J]. Journal of Xihua University (Natural Science Edition), 2010,29(2):223 226(to238).

[10]. Fan C.Y., Li S. C., Xu D.X., Similar Constructive Method of Solution for a Class of Boundary Value Problem of Thomson Equation (in Chinese) [J]. Advances in Applied Mathematics, 2012,1(2):41-48.

[11]. Li S. C., Liao Z.J., Constructing the Solution of Boundary Value Problem of the Differential Equation with its an Arbitrary Non-trivial Solution (in Chinese) [J]. Journal of Sichuan University (Natural Science Edition), 2012, 49(6):1209-1213.

[12]. Li S. C., Wu X.Q., The Similarity Structuring Method of Boundary Value Problems of the Composite Differential Equations (in Chinese) [J]. Journal of Xihua University (Natural science Edition), 2013,32(4):27-31.

[13]. Li S. C., The Similarity Structuring Method of Boundary Value Problems for Composite Differential Equations (in Chinese) [J]. Journal of Xihua University (Natural science Edition), 2013,32(1):23-26.

[14]. Li Q.Y., Li S.C., Li W., Wang J.C., Study of the Nonlinear Fluid Flow Modle in Dual-porosity Media Reservoir Based on Similar Structure (in Chinese) [J]. Chinese Journal of Engineering Mathematics, 2013, 30(1): 123-130.

[15]. Fan C.Y., Li S.C., Dong X.X., Bai L.X., Algorithms Design Based on Similar Structure (in Chinese) [J]. Advances in Applied Mathematics, 2013,2(3):107-113.

[16]. Dong X.X., Li S.C., Gui D.D., Pu J., Li H.C., Similar Constructing Method for Solving the Boundary Value Problem of the Composite First Weber system (in Chinese) [J]. American Journal of Applied Mathematics and Statistics, 2013,1(4),76-82.

[17]. Liu S.S., Liu S.D., Special Function [M]. Beijing: China Meteorological Press, 2002, 50-67. 